\title{
Estudio de los valores en el alumnado que cursa el grado de maestro en educación primaria en las universidades públicas de Andalucía
}

\author{
Gracia González-Gijón*, Nazaret Martínez-Heredia, Ana Amaro-Agudo y Andrés Soriano-Díaz \\ Universidad de Granada, Facultad de Ciencias de la Educación, Campus de Cartuja s/n, Granada - España \\ (e-mail: graciag@ugr.es; nazareth@ugr.es; anaamaro@ugr.es; asoriano@ugr.es)
}

* Autor a quién debe ser dirigida la correspondencia

Recibido Ago. 14, 2019; Aceptado Oct. 10, 2019; Versión final Nov. 7, 2019, Publicado Abr. 2020

\begin{abstract}
Resumen
La finalidad de este estudio es identificar y analizar los valores y en qué jerarquía se muestran en el alumnado que cursa estudios del grado de maestro en educación primaria en las Universidades públicas de la Comunidad Autónoma de Andalucía, España. Para ello, se ha empleado como instrumento de recogida el Test de valores adaptado (TVA adaptado) a una muestra de 651 estudiantes seleccionados mediante un muestreo probabilístico por conglomerados. Los resultados muestran una alta valoración hacia los aspectos no materiales relacionados con la afectividad, la moral, lo individual y la ecología, seguido de los valores relacionados con el cuerpo y su cuidado, lo social, lo material y lo estético; quedando al final valores relacionados con lo intelectual, lo político y lo religioso. Este estudio permite organizar el conocimiento acerca de los valores en los jóvenes universitarios, y realizar así una mejor aproximación a las necesidades educativas sobre la formación en valores en las carreras universitarias.
\end{abstract}

Palabras clave: valores; test; educación primaria; jóvenes universitarios; Andalucía (España).

\section{Study of the values present in the students pursuing the degree of teacher in primary education in the public universities of Andalucía}

\begin{abstract}
The objective of this research is to identify and analyze the values and in which hierarchy they are present in the students who are studying for the degree of teacher in primary education in the public universities of the Autonomous Community of Andalucía, Spain. For this, we have used as a collection instrument the Adapted Values Test (TVA adapted) to a sample of 651 students selected through a probabilistic sampling by clusters. The results show a high valuation towards non-material aspects related to affectivity, morality, individuality, and ecology, followed by values related to the body and its care. After these, they listed social, material and aesthetic concepts, leaving at the end values related to intellectual, political and religious issues. This study allows organizing the knowledge about values in young university students, making in this way a better approach to the educational needs about training in values in university careers.
\end{abstract}

Keywords: values; adapted value test; primary education; university students 


\section{INTRODUCCIÓN}

El actual siglo XXI ha traído consigo distintas perspectivas de interpretación de la realidad que han dado lugar a nuevas maneras de entenderla modificando los sistemas de relaciones humanas, sus fines y los medios. La sociedad española está experimentando rápidas y profundas transformaciones que tienen repercusiones importantes en la persona, en la cultura y en la educación. En realidad, el fundamento de tales transformaciones reside en un cambio del sistema de valores, por lo que en esta cambiante situación, es necesario llevar a cabo nuevas lecturas sobre las prácticas y los procesos educativos que se dan desde la perspectiva de los valores y de la educación en valores.

Los valores son un tema tan antiguo como de permanente actualidad como demuestran estudios recientes (Benedicto, 2017; García et al., 2017; González-Anleo y López-Ruiz, 2017; González-Gijón y Soriano, 2017; Maaranen et al., 2019; Van Krieken, 2019). Esta importancia del valor y de los valores en los seres humanos se ha traducido en multitud de definiciones (Marín, 1976; Reboul, 1999; Brady, 2011; Abramauskiené, 2015; Bhai y Horoi, 2019, etc.), acentuando cada una de ellas aspectos o dimensiones fundamentales de los mismos. En el presente estudio optamos por aquella que entiende que "el valor es una cualidad real o ideal, deseada o deseable por su bondad, cuya fuerza orienta la vida humana" (Gervilla, 2010).

En esta definición se manifiesta el sentido real e ideal del valor, así como su relación con la naturaleza humana. Los valores son deseados o deseables por su bondad, de tal manera que su fuerza estimativa orienta nuestra vida en una u otra dirección o finalidad, por ello van acompañados de un deber ser. La acción humana es siempre una acción moral por lo que cuando adoptamos valores estamos optando por lo que entendemos que es bueno o malo, por lo deseable o rechazable, las prioridades que se anteponen en la vida, los riesgos que se perciben y las esperanzas que depositamos en el futuro (Megías y Elzo, 2006). Valorar es dotar de sentido lo valorado y, por tanto, nombrar, distinguir, clasificar, introducir un orden, una jerarquía y un sentido (Sanmartín, 2005). Por todo ello, nuestras decisiones, acciones y prioridades están orientadas y dotadas de sentido a partir de un conjunto de valores individuales y sociales que guían nuestra conducta. De ahí que sea imposible una vida humana sin creencias ni valores, pues unas y otros son elementos constitutivos de nuestra personalidad, así como el fundamento de nuestras relaciones con los demás.

Junto a la definición de valor, es necesario clarificar también el concepto de persona del cual partimos, entendiéndola como "...animal de inteligencia emocional, singular y libre en sus decisiones, de naturaleza abierta o relacional, en el espacio y en el tiempo" (Gervilla, 2010). La dimensión animal genera los valores corporales, la inteligencia emocional nos conduce a los valores intelectuales y afectivos; de la singularidad y libertad surgen los valores morales, individuales y estéticos. Finalmente, de la naturaleza abierta o relacional, surgen los valores sociales, políticos, ecológicos, instrumentales y religiosos.

La finalidad del trabajo que se presenta es analizar las dimensiones descritas, en el futuro profesorado de educación primaria, por cuanto las personas que constituyen este colectivo serán los futuros maestros y maestras de enseñanza primaria, por ello, transmisores de valores que, en su labor docente, generarán un importante efecto axiológico multiplicador en su alumnado. El maestro, lo pretenda de manera consciente o no, es un transmisor de valores. Su modo de ser y de actuar puede ser, a veces, un modelo que emite un mensaje más poderoso que las palabras, por lo que cualquier actividad, hecho o acción que se produce en el aula, va tamizado por la forma en la que se muestra a su alumnado, de tal manera que no sólo enseña contenidos curriculares sino que él mismo es lo que enseña. La educación en valores se centra en desarrollar un ambiente pedagógico donde el aprendizaje vaya a las raíces mismas de los propios alumnos, se trata de reconocer que el crecimiento de una persona autónoma y responsable es prioritario en el ámbito moral (JaimeMirabal y Ladino-Luna, 2018).

Estos procesos no se dan únicamente de manera intencional sino que es en la dinámica escolar diaria donde se produce un mayor grado de influencia. Por ello, las experiencias cotidianas que se producen en la escuela son las que, sutilmente y de manera continua, van siendo interiorizadas por el alumnado, produciéndose así un proceso formativo integral indispensable para realizarse como personas y poder llevar a cabo el necesario crecimiento intelectual y moral. Además de lo anterior hay que señalar que el maestro: a) Selecciona, ordena y jerarquiza los contenidos que se imparten por lo que el currículum que llega al alumnado no es aséptico ni neutral. b) Presenta el contenido utilizando su metodología y enseña y evalúa según sus criterios. c) Activa el proceso de enseñanza-aprendizaje pudiendo inhibir, potenciar y sancionar según su criterio personal. d) Por la posición que ocupa y el poder que ostenta aparece como modelo por lo que los rasgos del mismo son susceptibles de imitación.

Investigar sobre los valores de los jóvenes que en un futuro próximo serán maestros es apostar por el conocimiento y por la construcción del futuro inmediato en lo referente a la formación docente, al modelo de escuela que queremos y al propio fundamento de la educación. El objetivo principal de nuestra investigación 
es describir y analizar la jerarquía de valores de los/as jóvenes andaluces que estudian el primer curso del grado de maestro en educación primaria así como explorar las diferencias existentes en relación al sexo.

\section{MÉTODOLOGIA}

Para la consecución del objetivo propuesto se comienza con una metodología descriptiva, de corte cuantitativo, empleando como instrumento de recogida de datos, un cuestionario. Esto se explica en las siguentes cuatro subsecciones: i) muestra y proceso de muestreo, ii) instrumento de recogida de información, iii) calidad del instrumento de recogida de información: y iv) procedimiento de recogida y análisis de datos

\section{Muestra y proceso de muestreo}

Nuestra investigación se realizó en los meses de mayo y junio del año 2018 en todas las universidades públicas de Andalucía, en concreto en las Facultades de Ciencias de la Educación. La muestra la formaron 651 estudiantes seleccionados mediante un muestreo probabilístico por conglomerados (ver tabla 1) donde la unidad primaria de muestreo fue el alumnado de todos los grupos de primer curso del Grado de maestro en Educación Primaria de las Facultades de Ciencias de la Educación de Andalucía, para posteriormente seleccionar de forma proporcional estos grupos en función del número de ellos en cada universidad andaluza (Arias-Gómez et al., 2016). De la muestra final 226 fueron hombres (34,7\%) y 425 mujeres (65,3\%) con edades comprendidas entre los 18 y 49 años. El grupo seleccionado de jóvenes adquiere una importancia singular, al tener un efecto multiplicador, pues al ser los educadores del futuro, van a transmitir a sus alumnos sus actitudes, creencias y valores.

Tabla 1: Muestro probabilístico por conglomerados

\begin{tabular}{|c|c|c|c|c|c|}
\hline \multicolumn{2}{|c|}{ Universidad } & \multirow{2}{*}{ 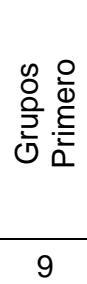 } & \multirow{2}{*}{ 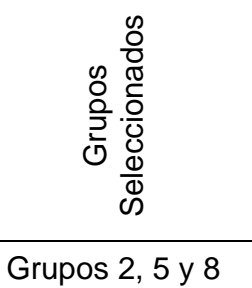 } & \multirow{2}{*}{ 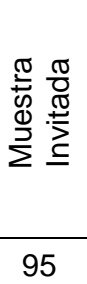 } & \multirow{2}{*}{ 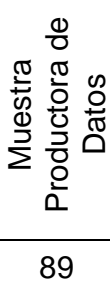 } \\
\hline \multirow{9}{*}{ 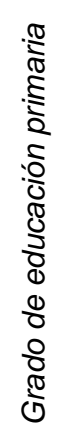 } & Sevilla & & & & \\
\hline & Granada & 8 & Grupos 1,2 y 3 & 180 & 159 \\
\hline & Almería & 3 & Grupo 3 & 50 & 40 \\
\hline & Córdoba & 4 & Grupos 1 y 2 & 85 & 82 \\
\hline & Huelva & 4 & Grupos 2 y 3 & 80 & 74 \\
\hline & Jaén & 4 & Grupos 3 y 4 & 130 & 128 \\
\hline & Cádiz & 3 & Grupo 1 & 20 & 16 \\
\hline & Málaga & 6 & Grupos 4 y 5,10 & 68 & 63 \\
\hline & \multicolumn{3}{|r|}{ Total } & \multicolumn{2}{|c|}{651} \\
\hline
\end{tabular}

\section{Instrumento de recogida de información}

El instrumento empleado ha sido el cuestionario denominado Test de Valores Adaptado (TVA_adaptado) que utiliza la técnica de calificación de palabras, agrupadas en 11 dimensiones o categorías que coinciden con los grupos de valores que mide: Corporales, intelectuales, afectivos, individuales, morales, estéticos, sociales, políticos, ecológicos, instrumentales y religiosos. Cada dimensión o categoría de valor incluye 25 palabras 0 conceptos que se valoraran por medio de una escala Likert (1 muy desagradable; 2 desagradable; 3 indiferente; 4 agradable y 5 muy agradable). Por tanto, el fundamento del Test reside en la reacción de agrado, desagrado o indiferencia que nos produce el leer o escuchar algunas palabras. Para la interpretación de los datos hay que tener en cuenta que para cada categoría de valor, la mayor puntuación positiva posible es 50 y la mayor puntuación negativa es -50 , por tanto, la reacción del sujeto será más favorable cuanto más se aproxime a 50 y más desfavorable cuanto más se aproxime a -50. El cero, así como las puntuaciones próximas a cero, pueden significar la indiferencia.

\section{Calidad del instrumento de recogida de información: validez y fiabilidad}

Como criterios de calidad del instrumento de recogida de información se utilizaron los parámetros de validez y de fiabilidad. Del primero, se tuvo en cuenta la validez de contenido mediante el Coeficiente de Concordancia W de Kendall, con las repuestas de 15 expertos. Se empleó para ello el programa estadístico SPSS-23. Los 
resultados mostraron el nivel de acuerdo entre las valoraciones de los jueces, obteniendo coeficientes próximos a 1. Con respecto a la fiabilidad, se midió la consistencia interna calculando el coeficiente alfa de Cronbach (1951). La puntuación alcanzada 0.972 muestra una alta consistencia interna de los ítems analizados y, por tanto, una alta fiabilidad.

\section{Procedimiento de recogida y análisis de datos}

Tras seleccionar los grupos mediante un muestreo probabilístico por conglomerados, se contactó con los/as docentes y se les informó de las características de la investigación y la duración del proceso de recogida de datos. Posteriormente se acordó el día y la hora para llevar a cabo dicho proceso en el que el equipo investigador se desplazó a cada provincia andaluza. La recogida de datos se iniciaba con una descripción sobre las características del test y su cumplimentación. Para el análisis de datos se utilizó el software estadístico IBM SPSS® en su versión 23.0 para Windows para realizar los análisis descriptivos básicos y pruebas $t$ de Student para grupos independientes. Previamente se procedió a la realización de la prueba Kolmogorov-Smirnov para verificar la distribución normal de las muestras.

\section{RESULTADOS}

Comenzamos presentando los principales resultados obtenidos de los estadísticos descriptivos analizados en este trabajo y que nos muestran la jerarquía de valores obtenida de los/as jóvenes participantes en este estudio (ver Tabla 2):

Tabla 2: Estadísticos descriptivos obtenidos por cada una de las categorías de valores

\begin{tabular}{|l|c|r|r|r|r|}
\hline \multicolumn{7}{|c|}{ Estadísticos descriptivos } \\
\hline & $\mathrm{N}$ & Mínimo & Máximo & Media & Desv. típ. \\
\hline C_Afectivos & 650 & -21 & 50 & 40,58 & 8,265 \\
\hline E_Morales & 650 & -34 & 50 & 38,65 & 9,834 \\
\hline D_Individuales & 650 & -10 & 50 & 37,29 & 9,766 \\
\hline I_Ecológicos & 650 & -25 & 50 & 34,93 & 12,038 \\
\hline A_Corporales & 650 & -17 & 50 & 33,68 & 8,005 \\
\hline G_Sociales & 647 & -16 & 50 & 30,53 & 11,010 \\
\hline J_Instrumentales & 650 & -26 & 50 & 24,42 & 11,227 \\
\hline F_Estéticos & 650 & -31 & 50 & 22,90 & 11,838 \\
\hline B_Intelectuales & 650 & -32 & 50 & 19,53 & 11,764 \\
\hline H_Políticos & 650 & -33 & 50 & 15,50 & 12,434 \\
\hline K_Religiosos & 649 & -50 & 50 & 6,64 & 21,093 \\
\hline
\end{tabular}

Los resultados descriptivos mostrados, y más concretamente las medias aritméticas, nos agrupan las categorías de valores en, aquellos que se encuentran por encima de 30, valores afectivos, morales, individuales, ecológicos, corporales y sociales, y los que se encuentran por debajo de 25 como son, los valores instrumentales, estéticos, intelectuales, políticos y religiosos. La categoría de valor que destaca por encima de las demás son los valores afectivos, con una media de 40.58. La originalidad de este test se encuentra en la valoración de 25 palabras en cada una de las categorías de valores, que nos permite detectar los diversos sentidos del valor, es decir, los aspectos que son más o menos valorados, o bien, rechazados. En dicha categoría encontramos, como palabras más valoradas, felicidad, madre, sentirse querido, familia y amistad. En último lugar aparece la palabra matrimonio. Cerca de los valores afectivos se sitúan los valores morales (media de 38.65) que incluye como palabra o ítem más valorado, el respeto, seguido de la libertad, la igualdad, verdad... el ítem menos valorado en los valores morales ha sido el deber. La categoría de valores individuales se encuentra muy próxima a la anterior (37.29) y en ella se priorizan palabras como superarse, generosidad, crecimiento personal, personalidad... y en último lugar, individualismo.

A continuación, aparecen las categorías de valores ecológicos (media de 34.93) donde encontramos en primer lugar el ítem aire puro, seguido de playa, sol, bosque... y en último lugar aparece gasolina sin plomo. Muy cerca están los valores corporales (33.68) que incluye, como palabras más valoradas, sonreír e higiene, seguidas de ducharse, vestir a mi gusto y dormir... y como menos valorada, maquillarse, categoría que además consiguió el mayor consenso por parte de los/as participantes con la desviación típica más baja (8.005). Para terminar este bloque de categorías de valores con una media por encima de 25, aparecen los valores sociales (30.53) con palabras más valoradas como igualdad de oportunidades, inclusión social, sociable y colaborar, inclusión social... y en menor promedio, popularidad. 
Dentro del grupo de valores que se encuentran por debajo de 25 están los valores instrumentales (24.42), formado por las palabras más valoradas vivienda y trabajo, seguidas de sueldo e internet... y menos valorado el ítem, apuestas. Le siguen muy de cerca la categoría de valores estéticos (con una media de 22.90) caracterizado por priorizar palabras como, música, puesta de sol, cine, opinión personal... y, en último lugar, la cirugía estética.

La siguiente categoría de valores son los intelectuales con una media de 19.53, que valora en los puestos con promedios más altos palabras como, enseñar, saber, aprender e inteligencia y con promedio más bajo, las conferencias. Los valores políticos también aparecen con una media cercana a la anterior, 15.50. Esta categoría de valores prioriza palabras como el bien común, plataforma stop desahucios, democracia, pacifismo... y el ítem menos valorado, dentro de los bajos promedios que presenta la categoría en sí, aparecen los partidos políticos. Mención especial merecen los resultados obtenidos por la categoría de valores religiosos que obtuvieron un promedio de 6.64 y con respecto al consenso de las valoraciones efectuadas, son los que menos acuerdo obtuvieron con una desviación típica de 21.093. Las palabras más valoradas fueron, caridad, libertad religiosa, misionero, procesión... y la menos valorada obispo.

Tras el análisis global realizamos un análisis descriptivo e inferencial comparativo desde la variable de identificación sexo y las variables referidas a las 11 categorías de valores descritas en este trabajo, corporales, intelectuales, afectivos, individuales, morales, estéticos, sociales, políticos, ecológicos, instrumentales y religiosos. Para llevar a cabo esta tarea hemos procedido a la comparación de medias entre dos grupos independientes mediante el test $t$ de Student, asumiendo los supuestos previos de distribución normal. A continuación se muestran los resultados obtenidos al aplicar la prueba t para relacionar la variable sexo con las 11 categorías de valores (ver tabla 3).

Como podemos observar, las categorías de valores que han conseguido puntuaciones superiores a 0.05 son los valores intelectuales, políticos, ecológicos, instrumentales y religiosos y, por tanto, no existen diferencias significativas en relación al sexo. En el resto de categorías podemos afirmar que existen diferencias significativas entre hombres y mujeres y que coinciden con las medias más altas y próximas a 5 . Así mismo, los promedios obtenidos por cada nivel de la variable sexo, nos indican que las mujeres tienen medias aritméticas más altas que los hombres por lo que valoran con más intensidad los aspectos relacionados con los valores corporales, intelectuales, afectivos, estéticos, individuales, morales, sociales, políticos, ecológicos y religiosos. Para terminar, destacamos en los valores religiosos, el bajo promedio obtenido por los hombres en la valoración de dicha categoría, media de 5.24, y la baja media, aunque mayor, reflejada por las mujeres $0,7.37$ (ver tabla 4).

\section{DISCUSIÓN}

La alta estimación de los valores afectivos, morales e individuales manifiesta una juventud con una gran personalidad y muy preocupada por la justicia, la humanización y las relaciones sociales y afectivas. Esto coincide con otros estudios recientes realizados en esta línea (Benninga, 2006; Berkowitz y Bier, 2005; Elzo et al., 2014; García et al., 2017; González-Anleo y López-Ruiz, 2017; González-Gijón y Soriano, 2017). Incluye los rasgos de la personalidad, mediante los cuales se expresan los valores que los objetos y las personas tienen para el ser humano. Refleja la formación de las potencialidades funcionales del ser humano, sus facultades físicas, intelectuales y espirituales y tiene como objetivo la formación integral de la personalidad. Los valores ecológicos, corporales y sociales, también con valores superiores a la media, revelan la importancia que otorgan los futuros educadores a la ecología, al cuerpo y la salud y a las relaciones y problemas sociales como afirman Collier (2013) y Daher (2019) en sus respectivos estudios.

La puntuación de los valores instrumentales, estéticos e intelectuales, inferiores a la media, ponen de manifiesto unos jóvenes poco materialistas, poco interesados en el arte y la belleza. Y, lo más preocupante, poco interesados en la formación intelectual, tal como se imparte hoy, siendo ésta su actual y diaria actividad, tal y como señalan Baburkin y Lymarev (2016) y Krumrei-Mancuso (2017). Los valores políticos y religiosos, los últimos de la jerarquía, y a bastante distancia de los precedentes, sobre todo el valor religioso, muestran la despreocupación de los jóvenes por la política, y la indiferencia y rechazo a las cuestiones religiosas. La alta desviación típica del valor religioso es un dato bastante significativo relacionado con estudios como el de los autores Hill y Den Dulk (2013) y Black (2017).

Otros estudios, indicados anteriormente, publicados en la Fundación Santa María (2017), el Ministerio de Asuntos Sociales (2017), Centro Reina Sofía sobre Adolescencia y Juventud (2019), INJUVE (2016), etc. se orientan en este mismo sentido y coinciden en afirmar el descenso acelerado del valor religioso en la población general y de los jóvenes en particular (González, 2004, 2011; Elzo, 1999; Elzo y Megías, 2014; GonzálezAnleo y López-Ruiz, 2017; Benedicto, 2017). 
Tabla 3: Prueba t de student para cada categoría de valores en relación al sexo

\begin{tabular}{|c|c|c|c|c|c|}
\hline \multicolumn{6}{|c|}{ Prueba de muestras independientes } \\
\hline & & $\begin{array}{l}\text { Prueba de } \\
\text { Levene }\end{array}$ & \multicolumn{3}{|c|}{ Prueba T para la igualdad de medias } \\
\hline & & \multirow[t]{2}{*}{ Sig. } & \multirow{2}{*}{$\begin{array}{l}\text { Sig. } \\
\text { (bilatera } \\
\text { I) }\end{array}$} & \multicolumn{2}{|c|}{$\begin{array}{l}\text { 95\% Intervalo de confianza para la } \\
\text { diferencia }\end{array}$} \\
\hline & & & & Inferior & Superior \\
\hline \multirow[t]{2}{*}{ A_Corporales } & $\begin{array}{l}\text { Se han asumido } \\
\text { varianzas iguales }\end{array}$ & 0,001 & 0,000 & 1,058 & 3,635 \\
\hline & $\begin{array}{l}\text { No se han asumido } \\
\text { varianzas iguales }\end{array}$ & & 0,001 & 0,956 & 3,737 \\
\hline \multirow[t]{2}{*}{ B_Intelectuales } & $\begin{array}{l}\text { Se han asumido } \\
\text { varianzas iguales }\end{array}$ & 0,843 & 0,416 & $-1,119$ & 2,703 \\
\hline & $\begin{array}{l}\text { No se han asumido } \\
\text { varianzas iguales }\end{array}$ & & 0,411 & $-1,102$ & 2,687 \\
\hline \multirow[t]{2}{*}{ C_Afectivos } & $\begin{array}{l}\text { Se han asumido } \\
\text { varianzas iguales }\end{array}$ & 0,000 & 0,000 & 1,623 & 4,271 \\
\hline & $\begin{array}{l}\text { No se han asumido } \\
\text { varianzas iguales }\end{array}$ & & 0,000 & 1,467 & 4,426 \\
\hline \multirow[t]{2}{*}{ D_Individuales } & $\begin{array}{l}\text { Se han asumido } \\
\text { varianzas iguales }\end{array}$ & 0,011 & 0,000 & 1,534 & 4,672 \\
\hline & $\begin{array}{l}\text { No se han asumido } \\
\text { varianzas iguales }\end{array}$ & & 0,000 & 1,453 & 4,752 \\
\hline \multirow[t]{2}{*}{ E_Morales } & $\begin{array}{l}\text { Se han asumido } \\
\text { varianzas iguales }\end{array}$ & 0,001 & 0,000 & 1,836 & 4,989 \\
\hline & $\begin{array}{l}\text { No se han asumido } \\
\text { varianzas iguales }\end{array}$ & & 0,000 & 1,770 & 5,055 \\
\hline \multirow[t]{2}{*}{ F_Estéticos } & $\begin{array}{l}\text { Se han asumido } \\
\text { varianzas iguales }\end{array}$ & 0,020 & 0,000 & 5,127 & 8,822 \\
\hline & $\begin{array}{l}\text { No se han asumido } \\
\text { varianzas iguales }\end{array}$ & & 0,000 & 5,012 & 8,936 \\
\hline \multirow[t]{2}{*}{ G_Sociales } & $\begin{array}{l}\text { Se han asumido } \\
\text { varianzas iguales }\end{array}$ & 0,136 & 0,000 & 2,466 & 5,993 \\
\hline & $\begin{array}{l}\text { No se han asumido } \\
\text { varianzas iguales }\end{array}$ & & 0,000 & 2,413 & 6,047 \\
\hline \multirow[t]{2}{*}{ H_Políticos } & $\begin{array}{l}\text { Se han asumido } \\
\text { varianzas iguales }\end{array}$ & 0,147 & 0,705 & $-1,631$ & 2,411 \\
\hline & $\begin{array}{l}\text { No se han asumido } \\
\text { varianzas iguales }\end{array}$ & & 0,711 & $-1,677$ & 2,457 \\
\hline \multirow[t]{2}{*}{ I_Ecológicos } & $\begin{array}{l}\text { Se han asumido } \\
\text { varianzas iguales }\end{array}$ & 0,007 & 0,007 & 0,750 & 4,641 \\
\hline & $\begin{array}{l}\text { No se han asumido } \\
\text { varianzas iguales }\end{array}$ & & 0,009 & 0,677 & 4,714 \\
\hline \multirow[t]{2}{*}{ J_Instrumentales } & $\begin{array}{l}\text { Se han asumido } \\
\text { varianzas iguales }\end{array}$ & 0,000 & 0,756 & $-1,536$ & 2,114 \\
\hline & $\begin{array}{l}\text { No se han asumido } \\
\text { varianzas iguales }\end{array}$ & & 0,775 & $-1,694$ & 2,272 \\
\hline \multirow[t]{2}{*}{ K_Religiosos } & $\begin{array}{l}\text { Se han asumido } \\
\text { varianzas iguales }\end{array}$ & 0,237 & 0,222 & $-1,294$ & 5,557 \\
\hline & $\begin{array}{l}\text { No se han asumido } \\
\text { varianzas iguales }\end{array}$ & & 0,239 & $-1,423$ & 5,686 \\
\hline
\end{tabular}


Tabla 4: Estadísticos descriptivos de la categoría de identificación sexo

\begin{tabular}{|c|c|c|c|c|c|}
\hline \multicolumn{6}{|c|}{ Estadísticos de grupo } \\
\hline & Sexo & $\mathrm{N}$ & Media & Desviación típ. & Error típ. de la media \\
\hline \multirow[t]{2}{*}{ A_Corporales } & Mujer & 428 & 34,49 & 7,213 & 0,349 \\
\hline & Hombre & 222 & 32,14 & 9,167 & 0,615 \\
\hline \multirow[t]{2}{*}{ B_Intelectuales } & Mujer & 428 & 19,80 & 11,887 & 0,575 \\
\hline & Hombre & 222 & 19,01 & 11,531 & 0,774 \\
\hline \multirow[t]{2}{*}{ C_Afectivos } & Mujer & 428 & 41,58 & 7,000 & 0,338 \\
\hline & Hombre & 222 & 38,64 & 10,010 & 0,672 \\
\hline \multirow[t]{2}{*}{ D_Individuales } & Mujer & 428 & 38,35 & 9,114 & 0,441 \\
\hline & Hombre & 222 & 35,25 & 10,640 & 0,714 \\
\hline \multirow[t]{2}{*}{ E_Morales } & Mujer & 428 & 39,82 & 9,266 & 0,448 \\
\hline & Hombre & 222 & 36,41 & 10,508 & 0,705 \\
\hline \multirow[t]{2}{*}{ F_Estéticos } & Mujer & 428 & 25,29 & 10,586 & 0,512 \\
\hline & Hombre & 222 & 18,31 & 12,763 & 0,857 \\
\hline \multirow[t]{2}{*}{ G_Sociales } & Mujer & 426 & 31,97 & 10,486 & 0,508 \\
\hline & Hombre & 221 & 27,74 & 11,477 & 0,772 \\
\hline \multirow[t]{2}{*}{ H_Políticos } & Mujer & 428 & 15,64 & 12,141 & 0,587 \\
\hline & Hombre & 222 & 15,25 & 13,004 & 0,873 \\
\hline \multirow[t]{2}{*}{ I_Ecológicos } & Mujer & 428 & 35,85 & 11,495 & 0,556 \\
\hline & Hombre & 222 & 33,16 & 12,864 & 0,863 \\
\hline \multirow[t]{2}{*}{ J_Instrumentales } & Mujer & 428 & 24,52 & 10,105 & 0,488 \\
\hline & Hombre & 222 & 24,23 & 13,145 & 0,882 \\
\hline \multirow[t]{2}{*}{ K_Religiosos } & Mujer & 427 & 7,37 & 20,224 & 0,979 \\
\hline & Hombre & 222 & 5,24 & 22,652 & 1,520 \\
\hline
\end{tabular}

Al hilo de lo anterior, los resultados globales están relacionados con otros estudios realizados en el ámbito español como por ejemplo, los análisis llevados a cabo por Elexpuru et al. (2013) y Murga (2008) calificando los valores morales, afectivos e individuales con la mayor puntuación por parte de los jóvenes universitarios. Del mismo modo, Ramos, Rhea, Pla y Abreu (2017) destacan que los valores más demandados desde la sociedad y cuya formación debe ser potenciada desde la Pedagogía, son la afectividad (amor a los seres humanos junto a la preocupación del desarrollo pleno de todos), y la solidaridad (comprometerse en acción e idea con el bienestar de los demás) para valorar el alto grado de compañerismo. Con respecto a las diferencias en relación al sexo, éstas coinciden con los promedios más altos en las categorías, valores afectivos, morales, individuales, estéticos y sociales, siendo las mujeres las que indican medias más altas, y que coinciden con los datos del informe del Centro Reina Sofía sobre Adolescencia y Juventud (2019) y con otros estudios pertenecientes al contexto internacional como, Sanford (2015), Pamela (2016) y Singh (2017). En general los valores presentes en la vida de un hombre y una mujer se vincula con los sentidos que se le otorgan a la vida personal propia y con las elecciones que se realizan en el seno familiar, social y profesional, los valores se relacionan con el estilo de vida y con la cultura (Sandoval Manríquez, 2007).

Actualmente, se suele hablar de una crisis de valores, debido a la existencia de múltiples estilos de vida, hábitos sociales, nuevas prácticas y discursos acerca de los procesos educativos. En este sentido, la globalización, la sociedad del conocimiento, la diversidad, las innovaciones tecnológicas, la creciente desigualdad... ha avivado el debate de la educación en valores (Brady, 2011). Es por ello, que la presente investigación favorece el conocimiento de los valores que son priorizados por el alumnado del grado de maestro en educación primaria en Universidades andaluzas. La consideración de la jerarquía de valores existente permite conocerlos en la construcción de la sociedad.

Los resultados que hemos presentado nos muestran un perfil de este alumnado. Sin profundizar en las valoraciones de las palabras que forman las categorías de valores, los resultados nos muestran aquello que los futuros maestros y maestras valoran en mayor medida. Finalmente, dicho estudio ha permitido organizar el conocimiento acerca de los valores en los/as jóvenes universitarios, realizando una aproximación al diagnóstico de las necesidades educativas en relación a la formación en valores en el ámbito universitario de nuestros futuros maestros/as. 


\section{CONCLUSIONES}

De acuerdo al trabajo presentado y a los resultados obtenidos, se pueden plantear las siguientes conclusiones principales:

1.- Los resultados muestran una juventud que se caracteriza por una personalidad definida y muy preocupada por aspectos de la vida como es la justicia, la humanización y las relaciones sociales y afectivas, seguidamente se muestran preocupados por la ecología y los valores relacionados con el cuerpo y su cuidado.

2.- Presentan un perfil de jóvenes poco materialistas y escasamente interesados en el arte y la belleza y con un bajo interés en la formación intelectual lo cual es preocupante e invita a la reflexión. Finalmente encontramos una despreocupación por la política, así como indiferencia y rechazo ante los temas religiosos.

3.- Las diferencias en relación al sexo las encontramos en los valores afectivos, morales, individuales, estéticos y sociales, siendo las mujeres las que indican medias más altas.

4.- Identificar y analizar los valores del alumnado que cursa estudios del grado de maestro en educación primaria en las Universidades públicas de la Comunidad Autónoma de Andalucía, España, nos permitirá aproximarnos y reflexionar sobre una necesaria formación en valores en el currículum universitario de los futuros maestros y maestras.

\section{REFERENCIAS}

Abramauskienè, J., The Attitude of Future Primary School Teachers towards National Values, Pedagogika, 119(3) (2015). Arias-Gómez, J., Villasís-Keever, M.A. y Miranda-Novales, M.G., The research protocol III. Study population. Rev. Alerg. Mex., 63(2), 201-6 (2016).

Baburkin, S. A., Talanov, S.L. y Lymarev, A.V., Vision of the Future and Values of University students, European Journal of Natural History, 6, 125-127 (2016).

Benedicto, J., Informe Juventud en España 2016, INJUVE (2017).

Benninga, J.S. y otros tres autores, Character and academics: What good schools do, Phi Delta Kappan, 87(6) (2006).

Berkowitz, M. y Bier. M., The interpersonal roots of character education, Character psychology and character education, 268-285 (2005).

Bhai, M. y Horoi, I., Teacher characteristics and academic achievement, Applied Economics, 1-19 (2019).

Black, P., Christian beliefs and values in science and religious education: an essay to assist the work of teachers of both subjects, International Studies in Catholic Education, 9(2), 206-222 (2017).

Brady, L., Teacher values and relationship: Factors in values education, Australian Journal of Teacher Education, 36(2) (2011).

Collier, K.G., The Social Purposes of Education: Personal and Social Values in Education, SciVal Topic Prominence (2013).

Cronbach, L.J., Coefficient alpha and the internal structure of test. Psychometrika, 297-334 (1951).

Daher, W., Values in the mathematics classroom, Educational Philosophy and Theory, 1-16 (2009).

Elexpuru, I., Villardón, L. y Yániz, C., Identificación y desarrollo de valores en estudiantes universitarios. Revista de Educación, 362,186-216 (2013).

Elzo, J., Jóvenes españoles 99. Fundación Santa María-Ediciones S. M., Madrid (1999).

Elzo, J. y Megías, E. (Codirect.), Jóvenes y valores I. Un ensayo de tipología, FAD, Centro Reina Sofía sobre Adolescencia y Juventud y Fundación ONCE, Madrid (2014).

García, V.A., Barbero, F. y Muñoz, R., Evaluación de la jerarquía de los valores humanos de Schwartz en la adolescencia: diferencias de género e implicaciones educativas, Revista Brasileira de Educação, 22(68), 123-146 (2017).

Gervilla, E., Educar en la Postmodernidad, Dykinson, Madrid (2010).

González, J., Jóvenes 2000 y religión, Fundación Santa María-Ediciones S. M., Madrid (2004).

González, J., Jóvenes españoles 2010, Fundación Santa María-Ediciones S. M., Madrid (2011).

González-Anleo, J. y López-Ruiz, P., Jóvenes españoles entre dos siglos 1984-2017, Fundación SM, Madrid (2017).

Gonzalez-Gijón, G. y Soriano, A., A study of the individual values of Puerto Rican younth, Revista de Paz y Conflictos. 10(1), 231-257 (2017).

Hill, J.P. y Den Dulk, K.R., Religion, volunteering, and educational setting: The effect of youth schooling type on civic engagement, Journal for the Scientific Study of Religion, 52(1), 179-197 (2013). 
González-Anleo, J.M. y López-Ruiz, J.A., Informe Fundación Santa María. (2017).

Informe Ministerio de Asuntos Exteriores. (2017).

Informe sobre valores Centro Reina Sofía sobre Adolescencia y Juventud. (2019).

Jaime-Mirabal, G. M. y Ladino-Luna, D., El método científico como alternativa didáctica de educación en valores para Escuelas de Ingeniería. Formación universitaria, 11(5), 3-10 (2018).

Krumrei-Mancuso, E.J., Intellectual humility and prosocial values: Direct and mediated effects, The Journal of Positive Psychology ,12(1), 13-28 (2017).

Maaranen, K. y otros cuatro autores, Teacher education matters: Finnish teacher educators' concerns, beliefs, and values, European Journal of Teacher Education, 42(2), 211-227 (2019).

Malins, P., How inclusive is "inclusive education" in the Ontario elementary classroom?: Teachers talk about addressing diverse gender and sexual identities, Teaching and Teacher Education, 54, 128-138 (2016).

Marín, R., Valores, objetivos y actitudes en educación, Miñón, Valladolid (1976).

Megías, E. y Elzo, J., Jóvenes, valores y drogas, FAD, Madrid (2006).

Murga, M.Á., Percepciones, valores y actitudes ante el desarrollo sostenible. Revista Española de Pedagogía, LXVI, (240), 327-24, (2008).

Ramos, J.M. y otros tres autores, La Pedagogía como Ciencia para el Tratamiento de los Contenidos Generales del Proceso Educativo y la Formación de Valores, Formación universitaria, 10(6), 77-86 (2017).

Reboul, O., Los valores de la educación, Barcelona, Idea Universitaria (1999).

Sandoval Manríquez, M., Sociología de los valores y juventud. Última década, 15(27), 95-118 (2007).

Sanford, K. y otros tres autores, Gender issues in initial teacher education in Canada: a eesearch LENS, Handbook of Canadian Research in Initial Teacher Education, 261 (2015).

Sanmartín, R., Los valores en los que educamos, Antropología: horizontes educativos, Universidad de Granada, Granada, (2005).

Singh, N., A study of values among school teachers at secondary level in relation to their gender local and marital status, Universidad de Mewar, Vietnan (2017).

Van Krieken, J., Participatory pedagogy for values education in early childhood education, European Early Childhood Education Research Journal, 27(3), 420-431 (2019). 
